\title{
Which beta-shifts have a largest invariant measure?
}

\author{
Vasso Anagnostopoulou \& Oliver Jenkinson
}

\begin{abstract}
For a given beta-shift, the lexicographic order induces a partial order (known as first-order stochastic dominance) on the collection of its shift-invariant probability measures. We characterise those beta-shifts for which this partial order has a largest element. These beta-shifts are all of finite type, and their lexicographically largest point is a periodic sequence of a particular kind: it is Sturmian (i.e. its shift-orbit is combinatorially equivalent to a rotation) with weight-per-symbol either an integer, or equal to $p /(a p+1)$ for some $a, p \geq 1$, or equal to $A+p /(p+1)$ for some $p \geq 1, A \geq 2$. In these cases, the largest invariant measure is precisely the unique one supported by the shift-orbit of the lexicographically largest point in the beta-shift.
\end{abstract}

\section{Introduction}

Rényi [33] considered the representation of real numbers with respect to an arbitrary base $\beta>1$. These representations, so-called $\beta$-expansions, are generated by orbits of the beta-transformation $T_{\beta}: x \mapsto \beta x(\bmod 1)$. Investigation of the ergodic properties of betatransformations, and their relation to $\beta$-expansions, has been a fruitful area of research (see e.g. $[5,6, \mathbf{1 2}, \mathbf{1 7}, \mathbf{3 0}, \mathbf{3 3}, \mathbf{3 4}, \mathbf{3 5}, \mathbf{3 7}, \mathbf{3 8}]$ ). The present article is motivated by the following problem: of the many $T_{\beta}$-invariant Borel probability measures on $[0,1]$, which, if any, is furthest to the right?

To precisely formulate the notion of one probability measure being further to the right than another, we require the partial order of first-order stochastic dominance (see e.g. [1, Ch. 18.8]): if $X$ is compact and totally ordered, and $\mu, \nu$ are Borel probability measures on $X$, then $\nu$ (first-order stochastically) dominates $\mu$, written $\mu \prec \nu$, if $\mu(f) \leq \nu(f)$ for all increasing functions $f: X \rightarrow \mathbb{R}$. Equipping $X=[0,1]$ with its usual order, a $T_{\beta}$-invariant probability measure would then be furthest to the right if it dominated all other $T_{\beta}$-invariant probability measures.

In fact, it is easily seen that the set of $T_{\beta}$-invariant probability measures never contains one which is furthest to the right: for example when $\beta=2$, the set of $T_{\beta}$-invariant probability measures has a supremum, namely the Dirac measure concentrated at the point 1 , yet this measure is not $T_{\beta}$-invariant, since 1 is not a fixed point of $T_{\beta}$. This unsatisfactory solution, stemming from the non-compactness of the set of $T_{\beta}$-invariant probability measures, is easily remedied by working instead with the symbolic version ${ }^{1}$ of $T_{\beta}$, its so-called beta-shift.

The (one-sided) beta-shift $X_{\beta}$ is a subset of $\{0, \ldots,[\beta]\}^{\mathbb{N}}$, defined as the closure (with respect to the product topology) of the set of sequences arising as a $\beta$-expansion. The left shift map $\sigma: X_{\beta} \rightarrow X_{\beta}$, defined by $\sigma:\left(x_{n}\right)_{n=1}^{\infty} \mapsto\left(x_{n+1}\right)_{n=1}^{\infty}$, is a continuous surjection, so the set $\mathcal{M}_{X_{\beta}}$ of $\sigma$-invariant Borel probability measures on $X_{\beta}$ is weak-* compact (see [39,

\footnotetext{
${ }^{1}$ The beta-shift is also the symbolic version of any map of the form $T(x)=F(x)(\bmod 1)$ for a differentiable real-valued function $F$ with $F^{\prime}>1$, and $F(0)=0$. Therefore the results of this paper have natural interpretations in terms of invariant probability measures for such maps $T$.
} 
Thm. 6.10]). After equipping $X_{\beta}$ with the lexicographic order ${ }^{2}$, the set $\mathcal{M}_{X_{\beta}}$ can be partially ordered by first-order stochastic dominance.

We may then ask: which beta-shifts have a largest invariant measure? In other words, we wish to determine those $\beta>1$ such that the partially ordered set $\left(\mathcal{M}_{X_{\beta}}, \prec\right)$ contains an element $\nu$ satisfying $\mu \prec \nu$ for all $\mu \in \mathcal{M}_{X_{\beta}}$. If $\beta>1$ is an integer then $X_{\beta}^{+}:=(\beta-1, \beta-1, \ldots)$ is the largest point in the corresponding beta-shift $X_{\beta}$, and also a fixed point for $\sigma$, so the following obviously holds:

THEOREM 1.1. If $\beta>1$ is an integer then there exists a largest shift-invariant measure on the beta-shift $X_{\beta}$, namely the Dirac measure concentrated on the point $X_{\beta}^{+}:=(\beta-1, \beta-1, \ldots)$.

Perhaps surprisingly, there also exist non-integer values of $\beta$ such that $X_{\beta}$ has a largest invariant measure. The purpose of this article is to determine the set of such values $\beta$.

For $1<\beta<2$, the characterisation is as follows ${ }^{3}$ :

TheOREM 1.2. For $1<\beta<2$, the following are equivalent:

(i) The beta-shift $X_{\beta}$ has a largest shift-invariant measure.

(ii) The lexicographically largest element in $X_{\beta}$ is the periodic sequence given by repeating the length- $(a \pi+1)$ word $\left(10^{a-1}\right)^{\pi} 0$, for some integers $a, \pi \geq 1$.

(iii) $\beta$ is the dominant ${ }^{4}$ root of the polynomial $\zeta^{a \pi+1}-\sum_{i=0}^{\pi} \zeta^{i a}$, for some integers $a, \pi \geq 1$.

In case the above conditions are satisfied, the largest shift-invariant measure on $X_{\beta}$ is the unique one supported by the periodic shift-orbit generated by the largest element of $X_{\beta}$.

For $\beta>2$, the existence of a largest invariant measure on $X_{\beta}$ entails a more stringent restriction on the periodic word defining the largest point in $X_{\beta}$ :

THEOREM 1.3. For non-integer $\beta>2$, the following are equivalent:

(i) The beta-shift $X_{\beta}$ has a largest shift-invariant measure.

(ii) The lexicographically largest element in $X_{\beta}$ is the periodic sequence given by repeating the length- $(\pi+1)$ word $B^{\pi}(B-1)$, for some integer $\pi \geq 1$, where $B$ is the integer part of $\beta$. (iii) $\beta$ is the dominant root of the polynomial $\zeta^{\pi+1}-B \sum_{i=0}^{\pi} \zeta^{i}$, for some integers $\pi \geq 1$, $B \geq 2$.

In case the above conditions are satisfied, the largest shift-invariant measure on $X_{\beta}$ is the unique one supported by the periodic shift-orbit generated by the largest element of $X_{\beta}$.

REMARK 1.4.

(i) If $\beta$ satisfies the conditions of one of the above theorems then $X_{\beta}$ is in particular a subshift of finite type (see [6, pp. 136-138]).

(ii) The golden mean $\beta=(1+\sqrt{5}) / 2$ satisfies the condition of Theorem 1.2 for $a=\pi=1$, so $X_{(1+\sqrt{5}) / 2}$ has a largest invariant measure, namely the one supported by its unique period-2 shift-orbit. More generally, if $\beta$ is a so-called multinacci number (cf. [29]), i.e. the dominant root of $\zeta^{\pi+1}-\sum_{i=0}^{\pi} \zeta^{i}$ for some $\pi \geq 1$, then Theorem 1.2 implies that $X_{\beta}$ has a largest invariant measure.

(iii) If $\beta \approx 2.65897$, the dominant root of $\zeta^{3}-2 \zeta^{2}-\zeta-2$, then $X_{\beta}$ does not have a largest invarant measure, by Theorem 1.3, since the lexicographically largest element of $X_{\beta}$ is the period-3 sequence $X_{\beta}^{+}:=211211 \ldots$, and 211 is not of the form $2^{\pi} 1$. As a direct verification of the absence of a largest invariant measure, note that $\left(\delta_{X_{\beta}^{+}}+\delta_{\sigma X_{\beta}^{+}}+\delta_{\sigma^{2} X_{\beta}^{+}}\right) / 3$ gives strictly largest integral to the increasing function $\chi_{\left\{X_{\beta}^{+}\right\}}$(the indicator function of $\left\{X_{\beta}^{+}\right\}$), but does not dominate the invariant probability measure supported by the period-2 orbit $\{0202 \ldots, 2020 \ldots\}$, since the latter measure gives larger integral to (for example) the indicator function for the set of points greater than or equal to $2020 \ldots$..

\footnotetext{
${ }^{2}$ This order is compatible with the one on [0,1]: if $\left(x_{i}\right)_{i=1}^{\infty}<\left(y_{i}\right)_{i=1}^{\infty}$ then $\sum_{i=1}^{\infty} x_{i} \beta^{-i}<\sum_{i=1}^{\infty} y_{i} \beta^{-i}$.

${ }^{3}$ In Theorems 1.2 and 1.3, $w^{k}$ denotes $k$-fold concatenation of the word $w$, cf. Section 2.1 .

${ }^{4} \mathrm{By}$ the dominant root we mean the largest real root.
} 
(iv) The dominant root of $\zeta^{4}-\zeta^{3}-\zeta^{2}-1$ is $\beta \approx 1.75488$. The largest element of $X_{\beta}$ is $X_{\beta}^{+}:=11001100 \ldots$, but 1100 is not of the form $\left(10^{a-1}\right)^{\pi} 0$, so Theorem 1.2 implies that $X_{\beta}$ does not have a largest invarant measure. This can be directly verified by showing (as in (iii) above) that the invariant measure supported by the orbit of $X_{\beta}^{+}$is not dominated by any other invariant measure, but on the other hand does not dominate the invariant measure supported by the period- 2 orbit $\{0101 \ldots, 1010 \ldots\}$.

(v) Comparison of (iii) and (iv) above gives some insight into the difference between Theorems 1.2 and 1.3: when $\beta>2$, the luxury of an alphabet containing at least 3 symbols makes it is easier to find invariant measures $\mu$ not dominated by the largest element of $X_{\beta}$.

REMARK 1.5. Given a bounded measurable function $f: X_{\beta} \rightarrow \mathbb{R}$, a measure $\nu \in \mathcal{M}_{X_{\beta}}$ is called $f$-maximizing (see e.g. $[\mathbf{3}, \mathbf{7}, \mathbf{2 1}])$ if $\mu(f) \leq \nu(f)$ for all $\mu \in \mathcal{M}_{X_{\beta}}$. So a largest invariant measure is one which is simultaneously $f$-maximizing for every increasing $f: X_{\beta} \rightarrow \mathbb{R}$.

For a comparable context see $[\mathbf{2 2}, \mathbf{2 3}]$, where certain invariant measures are shown to simultaneously optimize the integral of all convex functions (the associated partial order is known as second-order stochastic dominance, or alternatively as majorization). The optimizing measures in this case, so-called Sturmian ${ }^{5}$ measures (see also Section 3), appear to be rather ubiquitous in certain problems of ergodic optimization (see also $[\mathbf{3}, \mathbf{7}, \mathbf{8}, \mathbf{1 8}, \mathbf{1 9}]$ ).

In fact in the present paper, the largest invariant measures of Theorems 1.1-1.3 are also Sturmian: these measures are supported by periodic orbits whose permutation (under the shift map) is a rotation. However, unlike in $[\mathbf{3}, \mathbf{7}, \mathbf{8}, \mathbf{1 8}, \mathbf{1 9}, \mathbf{2 2}, \mathbf{2 3}]$, not all rotation numbers play a role; rather, the relevant rotation numbers are those rationals of the form $\pi /(a \pi+1)$ for integers $a, \pi \geq 1$.

Recall that an algebraic integer strictly larger than 1 is called a Pisot number if all of its algebraic conjugates have modulus strictly less than one. Among those $\beta>1$ such that $X_{\beta}$ has a largest invariant measure, the following result characterises those which are Pisot:

COROLlary 1.6. Let $\beta>1$ be non-integer, and such that $X_{\beta}$ has a largest invariant measure. The following are equivalent:

(i) $\beta$ is a Pisot number.

(ii) $\beta$ is the dominant root of $\zeta^{a \pi+1}-B \sum_{i=0}^{\pi} \zeta^{i a}$ for $(\pi, a, B)$ equal to either $(1,4,1)$, or $(1,3,1)$, or $(2,3,1)$, or $a=1$ and $\pi, B \geq 1$, or $(a, B)=(2,1)$ and $\pi \geq 1$.

(iii) $X_{\beta}$ is of Sturmian type ${ }^{6}$, and the weight-per-symbol of its Sturmian measure is either $1 / 5,1 / 4$, or $2 / 7$, or $B-1+\pi /(\pi+1)$ for integers $\pi, B \geq 1$, or $\pi /(2 \pi+1)$ for some integer $\pi \geq 1$.

The organisation of this article is as follows. Section 2 consists of preliminaries on symbolic dynamics, first-order stochastic dominance, and beta-shifts. Section 3 is devoted to Sturmian measures, and beta-shifts of Sturmian type, and includes the proof of Corollary 1.6. Theorems 1.1-1.3 are proved in Sections 4 and 5: beta-shifts without a largest invariant measure are dealt with in Theorem 4.5, while Theorem 5.4 pertains to beta-shifts which do have a largest invariant measure.

\section{Preliminaries}

\subsection{Symbolic dynamics.}

Notation 2.1. Let $\mathbb{N}$ denote the set of strictly positive integers, and set $\mathbb{N}_{0}:=\mathbb{N} \cup\{0\}$. For $m, n \in \mathbb{N}_{0}$, define $[m, n]:=\left\{i \in \mathbb{N}_{0}: m \leq i \leq n\right\}$.

\footnotetext{
${ }^{5}$ Some authors prefer the term balanced, cf. Remark 3.6.

${ }^{6}$ See Section 3 for the definitions of Sturmian type and weight-per-symbol.
} 
DeFinition 2.2. (Order, intervals, topology)

Define $F:=\mathbb{N}_{0}^{\mathbb{N}}$, the set of all sequences $x=\left(x_{n}\right)_{n=1}^{\infty}$, where $x_{n} \in \mathbb{N}_{0}$ for all $n \in \mathbb{N}$. For $x, x^{\prime} \in F$, we write $x<x^{\prime}$ if there exists $N \in \mathbb{N}$ with $x_{N}<x_{N}^{\prime}$ and $x_{n}=x_{n}^{\prime}$ for $1 \leq n<N$; we write $x \leq x^{\prime}$ when $x=x^{\prime}$ or $x<x^{\prime}$. This lexicographic order $\leq$ is a total order on $F$. Throughout this article the set $F$, and certain of its subsets, will always be equipped with the order $\leq$; in particular, whenever we say that some sequence is less than, or greater than, another sequence, this will always be with respect to the lexicographic order.

For $X \subset F$ and $x, x^{\prime} \in X$, define the closed interval $\left[x, x^{\prime}\right]_{X}:=\left\{y \in X: x \leq y \leq x^{\prime}\right\}$, and the half-open interval $\left(x, x^{\prime}\right]_{X}:=\left\{y \in X: x<y \leq x^{\prime}\right\}$. If the subset $X$ is clear from the context then these intervals may be denoted, respectively, by $\left[x, x^{\prime}\right]$ and $\left(x, x^{\prime}\right]$.

The set $F=\mathbb{N}_{0}^{\mathbb{N}}$ will always be equipped with the product topology. The shift map $\sigma: F \rightarrow F$, defined by $(\sigma x)_{n}=x_{n+1}$ for $n \in \mathbb{N}$, is then continuous.

DEFINITION 2.3. (Words)

A word is any element of the set $F \cup \bigcup_{n=0}^{\infty} \mathbb{N}_{0}^{n}$ (by convention the empty word $\varepsilon$ is the unique member of $\mathbb{N}_{0}^{0}$ ). By a finite word we mean an element of $\mathbb{N}_{0}^{*}=\bigcup_{n=0}^{\infty} \mathbb{N}_{0}^{n}$. It is usually convenient to suppress commas and braces, and write the finite word $w=\left(w_{1}, \ldots, w_{n}\right) \in \mathbb{N}_{0}^{n}$ as $w_{1} \ldots w_{n}$. The length of $w$, denoted $|w|$, is $n$, while its weight is defined to be $w_{1}+\ldots+w_{n}$. Its weight-per-symbol, is defined to be $\frac{1}{n}\left(w_{1}+\ldots+w_{n}\right)$.

Elements of $F$ are called infinite words or sequences. For each $n \in \mathbb{N}$, define $\pi_{n}: F \rightarrow \mathbb{N}_{0}^{n}$ by $\pi_{n}(x)=\left(x_{1}, \ldots, x_{n}\right)=x_{1} \ldots x_{n}$. A length- $m$ word $w$ is said to be a factor of the infinite word $x=\left(x_{i}\right)_{i=1}^{\infty}$ if $w=x_{j} x_{j+1} \ldots x_{j+m-1}$ for some $j \in \mathbb{N}$.

A sequence $x \in F$ is called periodic if there exists $p \in \mathbb{N}$ such that $x_{n}=x_{n+p}$ for all $n \in \mathbb{N}$; in this case the smallest such $p$ is called the period of $x$, and $\pi_{p}(x)$ is called the corresponding periodic word. Conversely, given a finite word $w=w_{1} \ldots w_{p}$, the periodic sequence determined by $w$, denoted $\bar{w}$, is the sequence $x=\left(x_{n}\right)_{n=1}^{\infty}$ such that $x_{n}=w_{n}(\bmod p)$ for all $n \in \mathbb{N}$. The periodic orbit determined by $w$ is the orbit $\left\{\sigma^{i}(\bar{w}): 0 \leq i \leq p-1\right\}$.

DeFinition 2.4. (Concatenation)

For a finite word $w$, and any word $x$, their concatenation $w x$ is the word defined by $(w x)_{i}=w_{i}$ for $i \in[1,|w|]$, and $(w x)_{i}=x_{i-|w|}$ for $i>|w|$. Under concatenation, the set $\mathbb{N}_{0}^{*}$ is the free monoid on $\mathbb{N}_{0}$, with $\varepsilon$ as its identity element; $\mathbb{N}_{0}^{*} \backslash\{\varepsilon\}$ is the free semi-group on $\mathbb{N}_{0}$.

Notation 2.5. If $w \in \mathbb{N}_{0}^{*}, n \in \mathbb{N}, G \subset F$, define $w^{n}:=\pi_{n|w|}(\bar{w})$ and $w G:=\{w x: x \in G\}$.

DeFinition 2.6. (Subshifts)

For any $k \in \mathbb{N}$, define $F_{k}:=\{0, \ldots, k-1\}^{\mathbb{N}}$. Clearly $\left(F_{k}\right)_{k=1}^{\infty}$ is an increasing sequence of sets, with $\bigcup_{k=1}^{\infty} F_{k}=l^{\infty}\left(\mathbb{N}_{0}\right)$, the set of all bounded sequences with entries in $\mathbb{N}_{0}$. When equipped with the product topology, each $F_{k}$ is compact. Clearly, the smallest element in $\left(F_{k}, \leq\right)$ is $\overline{0}=(0,0,0 \ldots)$, and the largest element is $\overline{k-1}=(k-1, k-1, k-1 \ldots)$.

Any non-empty closed subset $X \subset F_{k}$ satisfying $\sigma(X)=X$ is called a subshift (of $F_{k}$ ). For any subshift $X$ the restricted shift map $\left.\sigma\right|_{X}$ is a continuous endomorphism of $X$.

Notation 2.7. (Largest element)

If $X$ is any subshift then $(X, \leq)$ has a largest element, denoted $X^{+}$.

If $X^{+}$is periodic, let $\zeta_{X}$ denote its periodic word.

Define $m_{X}:=\pi_{1}\left(X^{+}\right) \in \mathbb{N}_{0}$, the largest integer appearing as an entry in elements of $X$.

Any interval in $X$ whose right endpoint is $X^{+}$is called an upper interval in $X$.

Notation 2.8. (Cylinder set, invariant measures)

For a subshift $X$, and $w \in \mathbb{N}_{0}^{*}$, define $\langle w\rangle_{X}:=\left\{x \in X: \pi_{|w|}(x)=w\right\}$, the associated cylinder set (and in particular a closed interval in $X$ ).

Let $\mathcal{M}_{X}$ denote the collection of $\sigma$-invariant Borel probability measures on $X$. 


\subsection{Dominance.}

Definition 2.9. For $X$ a subshift, and $\mu, \nu \in \mathcal{M}_{X}$, we say that $\mu$ is dominated by $\nu$ (or $\nu$ dominates $\mu$ ), and write $\mu \prec \nu$, if $\mu(f) \leq \nu(f)$ for every increasing ${ }^{7}$ function $f: X \rightarrow \mathbb{R}$.

It is easily shown that $\prec$ defines a partial order on $\mathcal{M}_{X}$, usually called first-order stochastic dominance. We use abbreviated terminology here because higher-order stochastic dominance will not be considered. See $[\mathbf{2 2}, \mathbf{2 3}]$ for usage of second-order stochastic dominance (also variously known as majorization, dilation, or balayage) in an ergodic theory setting.

Simple approximation arguments (cf. e.g. [25] or [27, Prop. 17.A.1]) yield the following useful reformulations of dominance:

Lemma 2.10. For $X$ a subshift, and $\mu, \nu \in \mathcal{M}_{X}$, the following are equivalent:

(i) $\mu \prec \nu$,

(ii) $\mu\left(x, X^{+}\right]_{X} \leq \nu\left(x, X^{+}\right]_{X}$ for all $x \in X$,

(ii) $\mu\left[x, X^{+}\right]_{X} \leq \nu\left[x, X^{+}\right]_{X}$ for all $x \in X$.

Definition 2.11. Let $X$ be a subshift. A measure $\mu \in \mathcal{M}_{X}$ is said to be the largest (respectively smallest) invariant measure on $X$ if it is the largest (respectively smallest) element in $\left(\mathcal{M}_{X}, \prec\right)$, i.e. if $\nu \prec \mu$ (respectively $\left.\mu \prec \nu\right)$ for all $\nu \in \mathcal{M}_{X}$.

REMARK 2.12.

(a) A subshift $X$ need not have either a largest or smallest invariant measure. Each $F_{k}$, however, has both a largest and a smallest invariant measure, namely the Dirac measures concentrated on the fixed points $\overline{k-1}=(k-1, k-1, k-1, \ldots)$ and $\overline{0}=(0,0,0 \ldots)$.

(b) For a Borel subset $Y \subset X$, a corresponding measure of maximal hitting frequency is defined (cf. [20]) to be any measure $\nu \in \mathcal{M}_{X}$ satisfying $\nu(Y)=\sup _{\mu \in \mathcal{M}_{X}} \mu(Y)$. Lemma 2.10 implies that $\nu \in \mathcal{M}_{X}$ is a largest invariant measure on $X$ if and only if it is the measure of maximum hitting frequency for each upper interval in $X$.

\subsection{Beta-shifts.}

Definition 2.13. For $x \in F$, the corresponding (one-sided) beta-shift is defined to be the largest closed, shift-invariant subset of $[\overline{0}, x]_{F}$, i.e. the set

$$
\bigcap_{n=0}^{\infty} \sigma^{-n}[\overline{0}, x]_{F} .
$$

Denote the collection of all beta-shifts by $\mathfrak{B}$.

REMARK 2.14.

(a) Distinct choices of $x$ in (1) may yield the same beta-shift $X$; clearly the smallest possible choice is $x=X^{+}$.

(b) Clearly, if $X, Y \in \mathfrak{B}$ then $X \subsetneq Y$ if and only if $X^{+}<Y^{+}$.

(c) Every beta-shift $X$ is contained in $F_{m_{X}}$, and hence in $F_{k}$ for every $k>m_{X}$.

(d) According to Definition 2.13, the singleton $\{\overline{0}\}$ is a beta-shift. All other beta-shifts are Cantor sets.

(e) By a result of Parry [30], Definition 2.13 is essentially ${ }^{8}$ equivalent to the definition of beta-shift mentioned in Section 1, in terms of $\beta$-expansions (see e.g. $[\mathbf{6}, \mathbf{3 0}, \mathbf{3 3}]$ ). Fixing a real number $\beta>1$, for each $t \in[0,1)$ define $d_{n}^{\beta}(t):=\left[\beta T_{\beta}^{n-1}(t)\right]$ for $n \geq 1$, where $T_{\beta}(t):=\beta t$ $(\bmod 1)$. The base- $\beta$ greedy expansion of $t$ is the sequence $d^{\beta}(t):=\left(d_{n}^{\beta}(t)\right)_{n=1}^{\infty} \subset F_{[\beta]}$. If $X_{\beta}$ denotes the closure in $F_{[\beta]}$ of $d^{\beta}([0,1))$, there is a unique $X \in \mathfrak{B}$ and $\beta>1$ such that $1=\sum_{i=1}^{\infty}\left(X^{+}\right)_{i} \beta^{-i}$, and hence $X_{\beta}=X$. Reciprocally, for every $X \in \mathfrak{B} \backslash\{\overline{0}\}$ there exists $\beta>1$ with $X_{\beta}=X$.

\footnotetext{
${ }^{7} f: X \rightarrow \mathbb{R}$ is increasing if $f(x) \leq f\left(x^{\prime}\right)$ whenever $x \leq x^{\prime}$.
}

${ }^{8}$ The only difference is our convention of including $\{\overline{0}\}$ as a beta-shift. 
(e) Every $X \in \mathfrak{B}$ contains the fixed point $\overline{0}$, and $\delta_{\overline{0}}$ is clearly the smallest element in $\left(\mathcal{M}_{X}, \prec\right)$. It is less obvious whether or not $\left(\mathcal{M}_{X}, \prec\right)$ has a largest element.

The following is immediate from Definition 2.13:

Lemma 2.15. Let $X \in \mathfrak{B}$, and $x \in F$. If $\sigma^{n}(x) \leq x \leq X^{+}$for all $n \geq 0$, then the orbit $\left\{\sigma^{n}(x): n \geq 0\right\}$ is contained in $X$.

Notation 2.16. For $X=\{\overline{0}\}$, define $X^{-}:=\overline{0}$. For $X \in \mathfrak{B} \backslash\{\overline{0}\}$, define $X^{-} \in X$ by

$$
\left(X^{-}\right)_{1}:=m_{X}-1 \text { and }\left(X^{-}\right)_{n}:=\left(X^{+}\right)_{n} \text { for } n \geq 2 \text {. }
$$

REMARK 2.17. For $X \in \mathfrak{B}$, each $x \in X$ has precisely one preimage ${ }^{9}$ in $\left(X^{-}, X^{+}\right]_{X}$ : if $x \in\left[\overline{0}, \sigma\left(X^{+}\right)\right]_{X}$ then $m_{X} x \in X$, while if $x \in\left(\sigma\left(X^{+}\right), X^{+}\right]_{X}$ then $\left(m_{X}-1\right) x \in X$.

Each $x \in\left[\overline{0}, \sigma\left(X^{+}\right)\right]_{X}$ has precisely $m_{X}+1$ preimages, while each $x \in\left(\sigma\left(X^{+}\right), X^{+}\right]_{X}$ has precisely $m_{X}$ preimages.

\section{Sturmian measures, and beta-shifts of Sturmian type}

Lemma 3.1. For $X \in \mathfrak{B}$, precisely one measure in $\mathcal{M}_{X}$ has its support contained in the interval $\left[X^{-}, X^{+}\right]_{X}$.

Proof. Existence is straightforward, since $\left[X^{-}, X^{+}\right]_{X}$ is a closed set whose shift image is $X$, while the proof of uniqueness is almost identical to that of Bousch \& Mairesse [8] for the case $X=X_{2}=F_{2}$ (cf. also $[\mathbf{9}]$ ).

Definition 3.2. A shift-invariant Borel probability measure on $F$ is called Sturmian if its support lies in $\left[X^{-}, X^{+}\right]_{X}$ for some $X \in \mathfrak{B}$. If we wish to emphasise the beta-shift $X$, we refer to the Sturmian measure for $X$, and denote it by $S_{X}$.

REMARK 3.3. The map $X \mapsto S_{X}$ on $\mathfrak{B}$ is not one-to-one: any Sturmian measure supported on a periodic orbit (with the exception of the fixed point $000 \ldots$...) is the Sturmian measure for (uncountably) many beta-shifts. For example the Dirac measure concentrated on $111 \ldots$ is the Sturmian measure for $X_{\beta}$ for all $2 \leq \beta \leq(3+\sqrt{5}) / 2$.

Definition 3.4. For a Sturmian measure $S_{X}$, call $\sum_{n \geq 1} n S_{X}\left(\langle n\rangle_{X}\right)$ its weight-per-symbol, and note that this equals

$$
\left(m_{X}-1\right) S_{X}\left(\left\langle m_{X}-1\right\rangle_{X}\right)+m_{X} S_{X}\left(\left\langle m_{X}\right\rangle_{X}\right)=S_{X}\left(\left\langle m_{X}\right\rangle_{X}\right)+m_{X}-1,
$$

because $\left[X^{-}, X^{+}\right]_{X} \subset\left\langle m_{X}-1\right\rangle_{X} \cup\left\langle m_{X}\right\rangle_{X}$.

The following standard properties of Sturmian measures will be required:

Proposition 3.5.

(i) For every $\varrho \in[0, \infty)$ there exists a unique Sturmian measure whose weight-per-symbol equals $\varrho$; we denote this measure by $S_{\varrho}$.

(ii) $S_{0}$ is the Dirac measure concentrated on the fixed point $000 \ldots$

(iii) For $\varrho \in(0,1)$, if $R_{\varrho}(t):=t+\varrho(\bmod 1)$, and

$$
x_{n}^{(\varrho)}(t):= \begin{cases}0 & \text { if } R_{\varrho}^{n-1}(t) \in[0,1-\varrho) \\ 1 & \text { otherwise },\end{cases}
$$

then $S_{\varrho}$ is the push forward of Lebesgue measure on $[0,1)$ under the map $t \mapsto\left(x_{n}^{(\varrho)}(t)\right)_{n=1}^{\infty}$. (iv) $S_{\varrho}=S_{\varrho-[\varrho]} \circ i_{[\varrho]}$ for $\varrho \in[1, \infty)$, where $i_{[\varrho]}$ is defined by $i_{[\varrho]}:\left(x_{n}\right)_{n=1}^{\infty} \mapsto\left(x_{n}-[\varrho]\right)_{n=1}^{\infty}$.

\footnotetext{
${ }^{9} \mathrm{~A}$ preimage always means a preimage under $\left.\sigma\right|_{X}$; so to say that $x$ has $l$ preimages means that $\left(\left.\sigma\right|_{X}\right)^{-1}(x)$ has cardinality $l$.
} 
(v) If $\varrho$ is irrational, the support of $S_{\varrho}$ is a uniquely ergodic Cantor set. If $\varrho$ is rational then $S_{\varrho}$ is supported on a single periodic orbit; writing $\varrho=A+\pi / q$, with $A \in \mathbb{N}_{0}, q \in \mathbb{N}$, $\pi \in[0, q-1]$, and $\operatorname{gcd}(\pi, q)=1$, if the points in this orbit are $s_{1}<\ldots<s_{q}$, then the shift $\sigma$ acts as a cyclic permutation:

$$
\sigma\left(s_{i}\right)= \begin{cases}s_{i+\pi} & \text { if } i \in[0, q-\pi] \\ s_{i+\pi-q} & \text { if } i \in[q-\pi+1, q],\end{cases}
$$

i.e. it is combinatorially equivalent to the action of $R_{\pi / q}(t):=t+\pi / q(\bmod 1)$ on any of its periodic orbits.

(vi) Any point in the support of a Sturmian measure is balanced: i.e. for each $a \in \mathbb{N}_{0}$, and all pairs of factors $u$ and $v$ of $x$ of equal length, $\left.|| u\right|_{a}-|v|_{a} \mid \leq 1$, where $|u|_{a}$ and $|v|_{a}$ denote the number of occurrences of $a$ in $u$ and $v$ respectively. If a periodic sequence is balanced, and contained in $\{A, A+1\}^{\mathbb{N}}$ for some $A \in \mathbb{N}_{0}$, then it is an atom of a (unique) Sturmian measure.

Proof. For (ii), observe that the Dirac measure $\delta_{\overline{0}}$ is the Sturmian measure for the beta-shift $\{\overline{0}\}$. For (iii), see e.g. $[\mathbf{3}, \mathbf{7}, \mathbf{8}, \mathbf{9}, \mathbf{2 2}, \mathbf{2 3}]$, though the connection with rotations goes back to [28]. For (iv), if $\tau \in[0,1)$ and $\operatorname{supp}\left(S_{\tau}\right) \subset\left[X^{-}, X^{+}\right]_{X}$ for some $X \in \mathfrak{B}$, then for any $A \in \mathbb{N}$, the support of $S_{\tau} \circ i_{A}$ lies in $\left[Y^{-}, Y^{+}\right]_{Y}$, where $Y \in \mathfrak{B}$ is defined by $\left(Y^{+}\right)_{n}=\left(X_{n}^{+}\right)_{n}+A$ for $n \in \mathbb{N}$. Property (i) then follows from existence and uniqueness of $S_{\varrho}$ for $\varrho \in[0,1)$ (see $[\mathbf{8}, \mathbf{9}]$ ), and (v) follows from (iii) and (iv) (cf. also $[\mathbf{3}, \mathbf{7}, \mathbf{8}, \mathbf{9}, \mathbf{2 2}, \mathbf{2 3}]$ ). The balanced property (vi) is well known, see e.g. [28] or [32, Ch. 6].

Remark 3.6. Sturmian measures appear explicitly in $[\mathbf{3}, \mathbf{7}, \mathbf{8}, \mathbf{1 8}, \mathbf{1 9}, \mathbf{2 2}, \mathbf{2 3}]$ in the case $\beta=2$, while the terminology Sturmian goes back to the description by Morse \& Hedlund $[\mathbf{2 8}]$ of the points in the support of Sturmian measures, so-called Sturmian sequences. ${ }^{10}$ For general $\beta>1$, Sturmian sequences are considered in $[\mathbf{1 0}, \mathbf{2 4}, \mathbf{2 6}]$.

Notation 3.7. For every $\varrho \in[0, \infty)$, there is clearly a unique $X(\varrho) \in \mathfrak{B}$ such that $X(\varrho)^{+}=\max \operatorname{supp}\left(S_{\varrho}\right) .{ }^{11}$ Any such beta-shift $X(\varrho)$ is said to be of Sturmian type.

If $\varrho \in[0, \infty)$ is rational, we use $\zeta(\varrho)$ as shorthand for $\zeta_{X(\varrho)}$, the periodic word for $X(\varrho)^{+}$.

Remark 3.8. If $X$ is a beta-shift of Sturmian type, and $S_{X}$ is periodic, then $X^{-}$is strictly pre-periodic, so the support of $S_{X}$ is contained in the half-open interval $\left(X^{-}, X^{+}\right]_{X}$.

The largest point in the support of a Sturmian measure can be generated by a concatenation procedure analogous to the Farey construction of rational numbers (see e.g. [15, Ch. III]). For $n \geq 1$, let $\mathfrak{F}_{n}$ denote the order- $n$ Farey sequence, i.e. the increasing finite sequence consisting of rationals in $[0,1]$ whose denominator is at most $n$, where 0 and 1 are included as $\frac{0}{1}$ and $\frac{1}{1}$ respectively. For example $\mathfrak{F}_{5}$ is $\frac{0}{0}, \frac{1}{5}, \frac{1}{4}, \frac{1}{3}, \frac{2}{5}, \frac{1}{2}, \frac{3}{5}, \frac{2}{3}, \frac{3}{4}, \frac{4}{5}, \frac{1}{1}$. Two rationals in $[0,1]$ are said to be Farey-consecutive if they appear as successive terms in some $\mathfrak{F}_{n}$.

Lemma 3.9. If $p_{1} / q_{1}<p_{2} / q_{2}$ (with $p_{1}, p_{2}, q_{1}, q_{2} \in \mathbb{N}, \operatorname{gcd}\left(p_{1}, q_{1}\right)=1=\operatorname{gcd}\left(p_{2}, q_{2}\right)$ ) are Farey-consecutive, then

$$
\zeta\left(\frac{p_{1}+p_{2}}{q_{1}+q_{2}}\right)=\zeta\left(\frac{p_{2}}{q_{2}}\right) \zeta\left(\frac{p_{1}}{q_{1}}\right) .
$$

If $\varrho \in[0,1]$ is irrational, and $\left\{\varrho_{i}\right\}$ is any sequence of rationals converging to $\varrho$, then $X(\varrho)^{+}$is the limit, in $F_{2}$, of $X\left(\varrho_{i}\right)^{+}$.

If $\varrho \in(1, \infty)$ then $X(\varrho)^{+}$is given by $\left(X(\varrho)^{+}\right)_{i}=\left(X(\varrho-[\varrho])^{+}\right)_{i}+[\varrho]$ for all $i \geq 1$.

Proof. The proof is easily adapted from the one in [31] (which treats the smallest point in the support of the Sturmian measure); see also e.g. [19].

\footnotetext{
${ }^{10}$ As indicated by Proposition 3.5 (vi), some authors prefer the term balanced, reserving the terminology Sturmian for the case of irrational $\varrho$ (see e.g. $[\mathbf{2}, \mathbf{4}, \mathbf{3 2}]$ ).

${ }^{11}$ Note that, with this notation, $S_{X(\varrho)}=S_{\varrho}$.
} 
Example 3.10. If $A \in \mathbb{N}_{0}$, and $B=A+1$, then $\zeta(A+1 / 2), \zeta(A+2 / 3), \zeta(A+3 / 5)$, $\zeta(A+5 / 8)$, and $\zeta(A+8 / 13)$ are, respectively,

$$
B A, B B A, B B A B A, B B A B B A B A, B B A B B A B A B B A B A,
$$

while

$$
X(A+(1+\sqrt{5}) / 2)^{+}=B B A B B A B A B B A B B A B A B B A B A B B A B B A B A B B A B A \ldots
$$

Notation 3.11. Let $\beta(\varrho)$ denote the unique $\beta>1$ which satisfies $X_{\beta}=X(\varrho)$.

The functions mapping $\varrho$ to $X(\varrho)^{+}, X(\varrho)$, and $\beta(\varrho)$ are all increasing:

Lemma 3.12. For $\varrho, \varrho^{\prime} \in[0, \infty)$,

$$
\varrho<\varrho^{\prime} \Longleftrightarrow X(\varrho)^{+}<X\left(\varrho^{\prime}\right)^{+} \Longleftrightarrow X(\varrho) \subsetneq X\left(\varrho^{\prime}\right) \Longleftrightarrow \beta(\varrho)<\beta\left(\varrho^{\prime}\right) .
$$

Proof. For the first equivalence, recall (cf. Notation 3.7) that the beta-shift $X(\tau)$ is defined by the equality $X(\tau)^{+}=\max \operatorname{supp}\left(S_{\tau}\right)$, and it is well known that $\max \operatorname{supp}\left(S_{\varrho}\right)<$ $\max \operatorname{supp}\left(S_{\varrho^{\prime}}\right)$ if and only if $\varrho<\varrho^{\prime}$ (see $[\mathbf{8}]$ or $\left.[\mathbf{9}]\right)$. The second equivalence is immediate from the definition of beta-shift (cf. Remark 2.14 (b)), while the third equivalence follows from the well known fact (see e.g. [6]) that $\beta<\beta^{\prime}$ if and only if $X_{\beta} \subsetneq X_{\beta^{\prime}}$.

We are now able to prove Corollary 1.6, which for convenience is re-stated here.

COROllary 1.6. Let $\beta>1$ be non-integer, and such that $X_{\beta}$ has a largest invariant measure. The following are equivalent:

(i) $\beta$ is a Pisot number.

(ii) $\beta$ is the dominant root of $\zeta^{a \pi+1}-B \sum_{i=0}^{\pi} \zeta^{i a}$ for $(\pi, a, B)$ equal to either $(1,4,1)$, or $(1,3,1)$, or $(2,3,1)$, or $a=1$ and $\pi, B \geq 1$, or $(a, B)=(2,1)$ and $\pi \geq 1$.

(iii) $X_{\beta}$ is of Sturmian type, and the weight-per-symbol of its Sturmian measure is either $1 / 5$, $1 / 4$, or $2 / 7$, or $B-1+\pi /(\pi+1)$ for integers $\pi, B \geq 1$, or $\pi /(2 \pi+1)$ for some integer $\pi \geq 1$.

Proof. Suppose $\beta>1$ is non-integer, and $X_{\beta}$ has a largest invariant measure. By Theorems 1.2 and $1.3, \beta=\beta(B-1+\pi /(a \pi+1))$ satisfies $\beta^{a \pi+1}=B \sum_{i=0}^{\pi} \beta^{i a}$, where either $B=1$ and $a, \pi \geq 1$, or $a=1$ and $\pi \geq 1, B \geq 2$.

If $a=1$ then $\beta^{\pi+1}=B \sum_{i=0}^{\pi} \beta^{i}$ for some $\pi, B \geq 1$, and [14, Thm. 2] implies that $\beta$ is Pisot. If $(a, B)=(2,1)$ then $0=\beta^{2 \pi+1}-\sum_{i=0}^{\pi} \beta^{2 i}=\beta^{2 \pi+1}-\frac{\beta^{2 \pi+2}-1}{\beta^{2}-1}$ for some $\pi \geq 1$, so [36, p. 597] implies that $\beta$ is Pisot.

The fourth smallest Pisot number is known to be $\theta_{4} \approx 1.4655$, the dominant root of $\theta^{3}-\theta^{2}-1$ (see $\left[\mathbf{1 3}\right.$, p. 120]). But this cubic polynomial is $\beta^{a \pi+1}-B \sum_{i=0}^{\pi} \beta^{i a}$ for $(\pi, a, B)=$ $(1,2,1)$, so in this case $\beta=\beta(1 / 3)=\theta_{4}$ is Pisot. If $a \geq 3$ then necessarily $B=1$, so $B-1+\frac{\pi}{a \pi+1}=\frac{\pi}{a \pi+1}<1 / 3$, so Lemma 3.12 gives $\beta=\beta\left(\frac{\pi}{a \pi+1}\right)<\beta(1 / 3)=\theta_{4}$, and therefore $\beta=\beta\left(\frac{\pi}{a \pi+1}\right)$ is not Pisot unless it is one of the three smallest Pisot numbers.

It turns out that the three smallest Pisot numbers are all of the form $\beta\left(\frac{\pi}{a \pi+1}\right)$ for some $a \geq 3, \pi \geq 1$. Siegel [36] proved that the smallest Pisot number is $\theta_{1} \approx 1.3247$, the dominant root of $\theta^{3}-\theta-1$, and that the second smallest is $\theta_{2} \approx 1.3802$, the dominant root of $\theta^{4}-\theta^{3}-1$. Dufresnoy \& Pisot [13, p. 120] proved that the third smallest Pisot number is $\theta_{3} \approx 1.4432$, the dominant root of $\theta^{5}-\theta^{4}-\theta^{3}+\theta^{2}-1$. If $(\pi, a)=(1,4)$ then $\beta=\beta\left(\frac{\pi}{a \pi+1}\right)=\beta(1 / 5)$ is the dominant root of $\beta^{5}-\beta^{4}-1=\left(\beta^{2}-\beta+1\right)\left(\beta^{3}-\beta-1\right)$, so $\beta=\beta(1 / 5)=\theta_{1}$ is Pisot. Similarly, if $(\pi, a)=(1,3)$ then $\beta=\beta(1 / 4)=\theta_{2}$ is Pisot, while if $(\pi, a)=(2,3)$ then $\beta=\beta(2 / 7)=\theta_{3}$ is Pisot.

There is the following formula for the mass given to a cylinder set by a Sturmian measure:

Lemma 3.13. Let $\varrho \in[0, \infty)$. If $w_{1} \ldots w_{n} \in \mathbb{N}_{0}^{n}$, and $c_{k}:=\sum_{i=1}^{k} w_{i}$ for $k \in[0, n]$, then

$$
S_{\varrho}\left(\left\langle w_{1} \ldots w_{n}\right\rangle_{F}\right)=\max \left(0,1+\min _{0 \leq k \leq n}\left(c_{k}-k \varrho\right)-\max _{0 \leq k \leq n}\left(c_{k}-k \varrho\right)\right) .
$$


Proof. The proof in [8, Prop. 1.2] is for $\varrho \in[0,1]$, and minor modifications yield (3) for $\varrho>1$.

REMARK 3.14. From Lemma 3.13, or alternatively by rearranging (2),

$$
S_{\varrho}\left(\left\langle m_{X(\varrho)}\right\rangle_{X(\varrho)}\right)=\varrho-\left(m_{X(\varrho)}-1\right),
$$

or in other words

$$
S_{\varrho}\left(\left\langle m_{X(\varrho)}\right\rangle_{X(\varrho)}\right)= \begin{cases}\varrho-[\varrho] & \text { if } \varrho \in[0, \infty) \backslash \mathbb{N}_{0}, \\ 1 & \text { if } \varrho \in \mathbb{N}_{0} .\end{cases}
$$

\section{Beta-shifts without a largest invariant measure}

The purpose of this section is to identify those beta-shifts which do not have a largest invariant measure. First, note that the Sturmian measure $S_{X}$ is the only candidate for a largest element of $\left(\mathcal{M}_{X}, \prec\right)$ :

Lemma 4.1. For $X \in \mathfrak{B}$, if $\mu\left(x, X^{+}\right]_{X}>S_{X}\left(x, X^{+}\right]_{X}$ for some $\mu \in \mathcal{M}_{X}, x \in X$, then $\left(\mathcal{M}_{X}, \prec\right)$ has no largest element.

Proof. By Lemma 3.1, if $\nu \in \mathcal{M}_{X} \backslash\left\{S_{X}\right\}$ then $\nu\left[X^{-}, X^{+}\right]_{X}<1=S_{X}\left[x^{\prime}, X\right]_{X}$. Therefore, by Lemma $2.10, S_{X}$ is the only possible largest element in $\left(\mathcal{M}_{X}, \prec\right)$. But $\mu\left(x, X^{+}\right]_{X}>$ $S_{X}\left(x, X^{+}\right]_{X}$, so $S_{X}$ is in fact not the largest element in $\left(\mathcal{M}_{X}, \prec\right)$.

Lemma 4.2. If $X \in \mathfrak{B}$ is not of Sturmian type, then $\left(\mathcal{M}_{X}, \prec\right)$ has no largest element.

Proof. Since $X$ is not of Sturmian type, and periodic points are dense in $X$ (see [37]), there exists a periodic point $x$ in the interval $\left(S_{X}^{+}, X^{+}\right]_{X}$, where $S_{X}^{+}$denotes the largest point in the support of $S_{X}$. If $\mu$ denotes the unique invariant probability measure whose support equals $\left\{\sigma^{i}(x): i \geq 0\right\}$, then $\mu\left(S_{X}^{+}, X^{+}\right]_{X}>0=S_{X}\left(S_{X}^{+}, X^{+}\right]_{X}$, so Lemma 4.1 implies that $\left(\mathcal{M}_{X}, \prec\right)$ has no largest element.

In view of Lemma 4.2, we may now restrict attention to those beta-shifts which are of Sturmian type.

Notation 4.3. Define

$$
U:=\left\{\frac{\pi}{a \pi+1}: a \in \mathbb{N}, \pi \in \mathbb{N}_{0}\right\} \cup\left\{A+\frac{\pi}{\pi+1}: A \in \mathbb{N}, \pi \in \mathbb{N}_{0}\right\} .
$$

REMARK 4.4. In continued fraction notation,

$$
U=\mathbb{N}_{0} \cup\left\{\left[0 ; c_{1}, c_{2}\right]: c_{1}, c_{2} \in \mathbb{N}\right\} \cup\left\{\left[c_{0} ; 1, c_{2}\right]: c_{0}, c_{2} \in \mathbb{N}\right\},
$$

where

$$
\left[c_{0} ; c_{1}, c_{2}\right]:=c_{0}+\frac{1}{c_{1}+\frac{1}{c_{2}}} \quad \text { for } c_{0}, c_{1}, c_{2} \in \mathbb{N} .
$$

The main result of this section is:

TheOrem 4.5. If $\varrho \notin U$ then $\left(\mathcal{M}_{X(\varrho)}, \prec\right)$ has no largest element.

To prove Theorem 4.5, it is convenient to define the set $V \supset U$ by

$$
V:=\left\{A+\frac{\pi}{a \pi+1}: A, \pi \in \mathbb{N}_{0}, a \in \mathbb{N}\right\} .
$$

We shall see that, if $\varrho \in V \backslash U$, the proof that $\left(\mathcal{M}_{X(\varrho)}, \prec\right)$ has no largest element is relatively straightforward. The heart of Theorem 4.5, therefore, is the case $\varrho \notin V$, and this will require considerable preparation. The strategy of proof is as follows.

By Lemma 4.1, it is sufficient to find a measure $\mu \in \mathcal{M}_{X(\varrho)}$ which is not dominated by the Sturmian measure $S_{\varrho}$. Although such a $\mu$ is not unique, some care is needed in its selection; 
in particular $\mu$ will depend on $\varrho$, so our first step will be to localise $\varrho$ with respect to the points in $V$. To this end we define, for each $A \in \mathbb{N}_{0}$,

$$
V_{A}:=\left\{A+\frac{\pi}{a \pi+1}: a, \pi \in \mathbb{N}\right\},
$$

and note that

$$
V=\bigcup_{A \in \mathbb{N}_{0}} V_{A} \cup\{A\}
$$

For $A \in \mathbb{N}_{0}$, we shall say that two elements $\varrho_{1}, \varrho_{2} \in V_{A}$, with $\varrho_{1}<\varrho_{2}$, are consecutive if $\left(\varrho_{1}, \varrho_{2}\right) \cap V_{A}=\emptyset$; equivalently, $\varrho_{1}=[A ; a, \pi]$ and $\varrho_{2}=[A ; a, \pi+1]$ for some $a, \pi \in \mathbb{N}$. Now each $\varrho \notin V$ lies in between two consecutive rationals in $V_{A}$, for some $A \in \mathbb{N}_{0}$. Letting $\varrho_{1}<\varrho_{2}$ denote these consecutive rationals, our next step will be to partition the open interval $\left(\varrho_{1}, \varrho_{2}\right)$ into infinitely many sub-intervals $R_{n}\left(\varrho_{1}, \varrho_{2}\right), 1 \leq n<\infty$ (see Definition 4.9). The choice of $\mu$ will depend on which of these sub-intervals contains $\varrho$. More precisely, for each $1 \leq n<\infty$ there is a shift-invariant probability measure $\mu=\mu_{n}$ (defined in Lemma 4.10) on $F$ which belongs to $\mathcal{M}_{X(\varrho)}$ for every $\varrho \in R_{n}\left(\varrho_{1}, \varrho_{2}\right)$, and is not dominated by $S_{\varrho}$.

To prove that $\mu$ is not dominated by $S_{\varrho}$, it is convenient to first introduce the following notation:

Notation 4.6. For $\varrho \in[0, \infty)$ and $\varrho^{\prime} \in[0, \infty) \cap \mathbb{Q}$, define

$$
C\left(\varrho^{\prime}, \varrho\right):=\left\langle\zeta\left(\varrho^{\prime}\right)\right\rangle_{X(\varrho)},
$$

the cylinder set in $X(\varrho)$ determined by $\zeta\left(\varrho^{\prime}\right)$, the periodic word of $X\left(\varrho^{\prime}\right)^{+}$.

For $\varrho \in V$, there is the following explicit formula for $\zeta(\varrho)$ :

Lemma 4.7. If $\varrho=A+\frac{\pi}{a \pi+1}$, with $A \in \mathbb{N}_{0}, a, \pi \in \mathbb{N}$, then $\zeta(\varrho)$ is the word

$$
\left(B A^{a-1}\right)^{\pi-1} B A^{a}=\left(B A^{a-1}\right)^{\pi} A,
$$

where $B=A+1$.

If $\varrho=A \in \mathbb{N}_{0}$ then $\zeta(\varrho)=A$.

Proof. If $\varrho=A \in \mathbb{N}$ then $X(\varrho)^{+}=\bar{A}$, so $\zeta(\varrho)=A$. Now suppose that $\varrho=A+\frac{\pi}{a \pi+1}$, with $A \in \mathbb{N}_{0}, a, \pi \in \mathbb{N}$. The length of the word $w$ defined by (5) is $a \pi+1$, and its weight is $(a \pi+1) A+\pi$, so its weight-per-symbol is $A+\frac{\pi}{a \pi+1}=\varrho$. It is readily verified that $\bar{w}$ is balanced, so by Proposition 3.5 (vi) it is an atom of the Sturmian measure $S_{\varrho}$. Moreover $\sigma^{n}(\bar{w}) \leq \bar{w}$ for all $n \geq 0$, so $X(\varrho)^{+}=\bar{w}$. Therefore $\zeta(\varrho)=w$, because there is no finite word $u$ such that $w=u^{n}$ for $n \geq 2$.

We will show (Lemma 4.10 and Corollary 4.12) that the measure $\mu=\mu_{n}$ (defined in Lemma 4.10) gives greater mass to the cylinder set $C\left(\varrho_{2}, \varrho\right)$ than does $S_{\varrho}$. By Lemma 2.10 this implies that $S_{\varrho}$ does not dominate $\mu$, since by the following result, $C\left(\varrho_{2}, \varrho\right)$ is an upper interval in $X(\varrho)$ :

Lemma 4.8. Let $A \in \mathbb{N}_{0}$, and suppose that $\varrho_{1}<\varrho_{2}$ are consecutive elements in $V_{A}$. For each $\varrho \in\left(\varrho_{1}, \varrho_{2}\right)$, the cylinder set $C\left(\varrho_{2}, \varrho\right)=\left\langle\zeta\left(\varrho_{2}\right)\right\rangle_{X(\varrho)}$ is an upper interval in the beta-shift $X(\varrho)$.

Proof. Immediate from Lemma 3.9

We can now define the sub-intervals $R_{n}\left(\varrho_{1}, \varrho_{2}\right)$ alluded to previously:

Definition 4.9. Suppose $\varrho_{1}<\varrho_{2}$ are consecutive elements in $V_{A}$, for some $A \in \mathbb{N}_{0}$, where $\varrho_{1}=p_{1} / q_{1}, \varrho_{2}=p_{2} / q_{2}$, and $p_{1}, p_{2}, q_{1}, q_{2} \in \mathbb{N}$, with $\operatorname{gcd}\left(p_{1}, q_{1}\right)=1=\operatorname{gcd}\left(p_{2}, q_{2}\right)$. For $n \in \mathbb{N}_{0}$, define

$$
r_{n}\left(\varrho_{1}, \varrho_{2}\right):=\frac{p_{2} q_{1} n+p_{1}}{q_{2} q_{1} n+q_{1}}
$$


and note that

$$
\zeta\left(r_{n}\left(\varrho_{1}, \varrho_{2}\right)\right)=\zeta\left(\varrho_{2}\right)^{q_{1} n} \zeta\left(\varrho_{1}\right)
$$

Define

$$
R_{1}\left(\varrho_{1}, \varrho_{2}\right):=\left(r_{0}\left(\varrho_{1}, \varrho_{2}\right), r_{1}\left(\varrho_{1}, \varrho_{2}\right)\right)=\left(\varrho_{1}, r_{1}\left(\varrho_{1}, \varrho_{2}\right)\right)
$$

and

$$
R_{n}\left(\varrho_{1}, \varrho_{2}\right):=\left[r_{n-1}\left(\varrho_{1}, \varrho_{2}\right), r_{n}\left(\varrho_{1}, \varrho_{2}\right)\right) \quad \text { for } n \geq 2 .
$$

Our strategy is to show that, for each $n \geq 1$, if $\varrho \in R_{n}\left(\varrho_{1}, \varrho_{2}\right)$ then the invariant measure $\mu$ supported on the periodic orbit defined by the word $\zeta\left(\varrho_{2}\right)^{n} A$ lies in $\mathcal{M}_{X(\varrho)}$, and gives larger mass to the upper interval $C\left(\varrho_{2}, \varrho\right)$ than does the Sturmian measure $S_{\varrho}$. The first step is the following:

LEMMA 4.10. Let $A \in \mathbb{N}_{0}$, and suppose that $\varrho_{1}<\varrho_{2}$ are consecutive elements in $V_{A}$. For all $n \geq 1$, and every $\varrho \in R_{n}\left(\varrho_{1}, \varrho_{2}\right)$, the periodic orbit defined by $\zeta\left(\varrho_{2}\right)^{n} A$ lies in $X(\varrho)$; consequently, the invariant measure $\mu_{n}$ carried by this periodic orbit belongs to $\mathcal{M}_{X(\varrho)}$.

If $\varrho_{2}=p_{2} / q_{2}$ where $p_{2}, q_{2} \in \mathbb{N}$, $\operatorname{gcd}\left(p_{2}, q_{2}\right)=1$, then

$$
\mu_{n}\left(C\left(\varrho_{2}, \varrho\right)\right)=\frac{n}{n q_{2}+1} .
$$

Proof. Let $\varrho_{1}=p_{1} / q_{1}$, where $p_{1}, q_{1} \in \mathbb{N}, \operatorname{gcd}\left(p_{1}, q_{1}\right)=1$, and suppose $\varrho \in R_{n}\left(\varrho_{1}, \varrho_{2}\right)$.

First consider the case $n=1$, so that $R_{n}\left(\varrho_{1}, \varrho_{2}\right)$ is the open interval $\left(\frac{p_{1}}{q_{1}}, \frac{p_{2} q_{1}+p_{1}}{q_{2} q_{1}+q_{1}}\right)$. If $\varrho \in R_{1}\left(\varrho_{1}, \varrho_{2}\right)$ then, by Lemma 3.9,

$$
X(\varrho)^{+}>\zeta\left(\varrho_{2}\right) \overline{\zeta\left(\varrho_{1}\right)}
$$

Moreover,

$$
\overline{\zeta\left(\varrho_{2}\right)^{n} A}=\overline{\zeta\left(\varrho_{2}\right) A}<\zeta\left(\varrho_{2}\right) \overline{\zeta\left(\varrho_{1}\right)}
$$

because

and Lemma 4.7 gives

$$
\left(\overline{\zeta\left(\varrho_{2}\right) A}\right)_{i}=\left(\zeta\left(\varrho_{2}\right) \overline{\zeta\left(\varrho_{1}\right)}\right)_{i} \quad \text { for } 1 \leq i \leq q_{2}
$$

$$
\left(\overline{\zeta\left(\varrho_{2}\right) A}\right)_{q_{2}+1}=A<B=\left(\zeta\left(\varrho_{2}\right) \overline{\zeta\left(\varrho_{1}\right)}\right)_{q_{2}+1} .
$$

Combining (7) and (8) gives

$$
\overline{\zeta\left(\varrho_{2}\right)^{n} A}<X(\varrho)^{+} .
$$

But $\overline{\zeta\left(\varrho_{2}\right)^{n} A}$ is the largest point in its orbit, so (9) and Lemma 2.15 together imply that this orbit is contained in $X(\varrho)$, as required.

Now suppose that $n \geq 2$. It is readily verified that $X\left(r_{n-1}\left(\varrho_{1}, \varrho_{2}\right)\right)^{+}=\overline{\zeta\left(\varrho_{2}\right)^{q_{1}(n-1)} \zeta\left(\varrho_{1}\right)}$. Since $\varrho \geq r_{n-1}\left(\varrho_{1}, \varrho_{2}\right)$, Lemma 3.12 gives

$$
X(\varrho)^{+} \geq X\left(r_{n-1}\left(\varrho_{1}, \varrho_{2}\right)\right)^{+}=\overline{\zeta\left(\varrho_{2}\right)^{q_{1}(n-1)} \zeta\left(\varrho_{1}\right)} .
$$

We claim that

$$
\overline{\zeta\left(\varrho_{2}\right)^{n} A}<\overline{\zeta\left(\varrho_{2}\right)^{q_{1}(n-1)} \zeta\left(\varrho_{1}\right)} .
$$

To prove (11), note that $\varrho_{1}=p_{1} / q_{1}$ is not an integer, so $q_{1} \geq 2$, and therefore $n \leq q_{1}(n-1)$. So the first $n q_{2}$ symbols of $\overline{\zeta\left(\varrho_{2}\right)^{q_{1}(n-1)} \zeta\left(\varrho_{1}\right)}$ coincide with the first $n q_{2}$ symbols of $\overline{\zeta\left(\varrho_{2}\right)^{n} A}$. Now the $\left(n q_{2}+1\right)$-st symbol of $\overline{\zeta\left(\varrho_{2}\right)^{q_{1}(n-1)} \zeta\left(\varrho_{1}\right)}$ is the first symbol of either $\zeta\left(\varrho_{2}\right)$ or $\zeta\left(\varrho_{1}\right)$, namely $B=A+1$ (by Lemma 4.7), thus

$$
\left(\overline{\zeta\left(\varrho_{2}\right)^{q_{1}(n-1)} \zeta\left(\varrho_{1}\right)}\right)_{n q_{2}+1}=A+1>A=\left(\overline{\zeta\left(\varrho_{2}\right)^{n} A}\right)_{n q_{2}+1},
$$

so indeed (11) holds. Combining with (10) gives $\overline{\zeta\left(\varrho_{2}\right)^{n} A}<X(\varrho)^{+}$, and because $\overline{\zeta\left(\varrho_{2}\right)^{n} A}$ is the largest point in its orbit, Lemma 2.15 implies that the whole of this orbit lies in $X(\varrho)$, as required. 
To prove (6) for $n \geq 1$ we must show that $\left\{1 \leq i \leq n q_{2}+1: \pi_{q_{2}}\left(\sigma^{i-1} x\right)=\zeta\left(\varrho_{2}\right)\right\}$ has cardinality $n$, where $x:=\overline{\zeta\left(\varrho_{2}\right)^{n} A}$. For this it suffices to check that $\zeta\left(\varrho_{2}\right)$ is not a sub-word of either $\alpha\left(\varrho_{2}\right) \omega\left(\varrho_{2}\right)$ or $\alpha\left(\varrho_{2}\right) A \omega\left(\varrho_{2}\right)$, where $\alpha\left(\varrho_{2}\right)$ (respectively $\omega\left(\varrho_{2}\right)$ ) denotes the length$\left(q_{2}-1\right)$ word obtained by deleting the first (respectively the last) symbol from the word $\zeta\left(\varrho_{2}\right)$; these facts are readily verified using the formula (5) from Lemma 4.7.

Lemma 4.11. Suppose $A \in \mathbb{N}_{0}$, and $\varrho_{1}=p_{1} / q_{1}<\varrho_{2}=p_{2} / q_{2}$ are consecutive elements in $V_{A}$, where $p_{1}, p_{2}, q_{1}, q_{2} \in \mathbb{N}$, and $\operatorname{gcd}\left(p_{1}, q_{1}\right)=1=\operatorname{gcd}\left(p_{2}, q_{2}\right)$. Then for all $\varrho \in\left[\varrho_{1}, \varrho_{2}\right]$,

$$
S_{\varrho}\left(C\left(\varrho_{2}, \varrho\right)\right)=q_{1} \varrho-p_{1},
$$

and in particular $\varrho \mapsto S_{\varrho}\left(C\left(\varrho_{2}, \varrho\right)\right)$ is strictly increasing on $\left[\varrho_{1}, \varrho_{2}\right]$.

Proof. Write $\varrho_{1}=A+\frac{\pi}{a \pi+1}$ and $\varrho_{2}=A+\frac{\pi+1}{a(\pi+1)+1}$, for $a, \pi \in \mathbb{N}$. In view of Lemma 3.13 , we wish to evaluate, for $\varrho \in\left[\varrho_{1}, \varrho_{2}\right]$, the expression

$$
1+\min _{0 \leq k \leq q_{2}}\left(c_{k}-k \varrho\right)-\max _{0 \leq k \leq q_{2}}\left(c_{k}-k \varrho\right),
$$

where

$$
c_{k}:=\sum_{i=1}^{k} \zeta\left(\varrho_{2}\right)_{i} \quad \text { for } k \in\left[0, q_{2}\right] .
$$

By Lemma 4.7, $\zeta\left(\varrho_{2}\right)=\left(B A^{a-1}\right)^{\pi} A$, where $B=A+1$. Therefore $c_{0}=0$,

$$
c_{k}=k A+i \quad \text { for } k \in[(i-1) a+1, i a], i \in[1, \pi+1],
$$

and

$$
c_{q_{2}}=c_{(\pi+1) a+1}=((\pi+1) a+1) A+\pi+1 .
$$

From (13) and (14) we see that, for $k \in\left[0, q_{2}\right]$, the minimum value of $c_{k}-k \varrho$ is attained when $k=j a$ for some $0 \leq j \leq \pi$. Therefore

$$
\min _{0 \leq k \leq q_{2}}\left(c_{k}-k \varrho\right)=\min _{0 \leq j \leq \pi} j(a A+1-a \varrho) .
$$

But $a A+1-a \varrho>0$ because $\varrho \leq \varrho_{2}=A+\frac{\pi+1}{a(\pi+1)+1}<A+1 / a$, so the minimum is attained when $j=0$, thus

$$
\min _{0 \leq k \leq q_{2}}\left(c_{k}-k \varrho\right)=0 .
$$

The maximum value of $c_{k}-k \varrho$ is attained when $k=j a+1$ for some $0 \leq j \leq \pi$, so

$$
\max _{0 \leq k \leq q_{2}}\left(c_{k}-k \varrho\right)=\max _{0 \leq j \leq \pi} j(a A+1-a \varrho)+A+1-\varrho=\pi(a A+1-a \varrho)+A+1-\varrho,
$$

again because $a A+1-a \varrho>0$. From (15) and (16), and since $p_{1} / q_{1}=A+\pi /(a \pi+1)$,

$$
1+\min _{0 \leq k \leq q_{2}}\left(c_{k}-k \varrho\right)-\max _{0 \leq k \leq q_{2}}\left(c_{k}-k \varrho\right)=\varrho(q \pi+1)-((a \pi+1) A+\pi)=q_{1} \varrho-p_{1},
$$

In particular, $q_{1} \varrho-p_{1} \geq 0$ for $\varrho \in\left[\varrho_{1}, \varrho_{2}\right]$, so (12) follows from Lemma 3.13.

Corollary 4.12. Suppose $A \in \mathbb{N}_{0}$, and $\varrho_{1}=p_{1} / q_{1}<\varrho_{2}=p_{2} / q_{2}$ are consecutive elements in $V_{A}$, where $p_{1}, p_{2}, q_{1}, q_{2} \in \mathbb{N}$, and $\operatorname{gcd}\left(p_{1}, q_{1}\right)=1=\operatorname{gcd}\left(p_{2}, q_{2}\right)$. For all $n \geq 1$, and $\varrho \in R_{n}\left(\varrho_{1}, \varrho_{2}\right)$,

$$
S_{\varrho}\left(C\left(\varrho_{2}, \varrho\right)\right)<S_{r_{n}\left(\varrho_{1}, \varrho_{2}\right)}\left(C\left(\varrho_{2}, r_{n}\left(\varrho_{1}, \varrho_{2}\right)\right)\right)=\frac{n}{n q_{2}+1}=\mu_{n}\left(C\left(\varrho_{2}, \varrho\right)\right) .
$$

Proof. By Lemma 4.11, the function $\varrho \mapsto S_{\varrho}\left(C\left(\varrho_{2}, \varrho\right)\right)$ is strictly increasing on the interval $\left[\varrho_{1}, \varrho_{2}\right]$, hence on each interval $R_{n}\left(\varrho_{1}, \varrho_{2}\right)$, for $n \geq 1$. Therefore, if $\varrho \in R_{n}\left(\varrho_{1}, \varrho_{2}\right)$ then $S_{\varrho}\left(C\left(\varrho_{2}, \varrho\right)\right)<S_{r_{n}\left(\varrho_{1}, \varrho_{2}\right)}\left(C\left(\varrho_{2}, r_{n}\left(\varrho_{1}, \varrho_{2}\right)\right)\right)$. After setting $\varrho=r_{n}\left(\varrho_{1}, \varrho_{2}\right)=\frac{p_{2} q_{1} n+p_{1}}{q_{2} q_{1} n+q_{1}}$ in (12), a short calculation gives $S_{r_{n}\left(\varrho_{1}, \varrho_{2}\right)}\left(C\left(\varrho_{2}, r_{n}\left(\varrho_{1}, \varrho_{2}\right)\right)\right)=\frac{n}{n q_{2}+1}$, and by $(6)$ this is also the value of $\mu_{n}\left(C\left(\varrho_{2}, \varrho\right)\right)$, so (17) is proved. 
Proof of Theorem 4.5. Each $\varrho \notin V$ belongs to $\left(\varrho_{1}, \varrho_{2}\right)$, where $\varrho_{1}<\varrho_{2}$ are consecutive elements in $V_{A}$ for some $A \in \mathbb{N}_{0}$. Now $C\left(\varrho, \varrho_{2}\right)$ is an upper interval in $X(\varrho)$ by Lemma 4.8, so the inequality (17) implies that $S_{\varrho}$ does not dominate $\mu_{n} \in \mathcal{M}_{X(\varrho)}$. Therefore, by Lemma 4.1, $\left(\mathcal{M}_{X(\varrho)}, \prec\right)$ has no largest element.

It remains to show that if $\varrho \in V \backslash U$ then $S_{\varrho}$ does not dominate every measure in $\mathcal{M}_{X(\varrho)}$. Now $\varrho \in V \backslash U$ implies firstly that $q=a \pi+1$ for $a \geq 2$, and secondly that $\varrho>1$, hence $m=m_{X(\varrho)} \geq 2$. Now $X(\varrho)^{+}$is a (Sturmian) sequence on the symbol set $\{m-1, m\}$, and belongs to $\langle m\rangle_{X(\varrho)}$, so both points $\overline{0 m}$ and $\overline{m 0}$ are smaller than $X(\varrho)^{+}$. Hence the period-2 orbit $\{\overline{0 m}, \overline{m 0}\}$ lies in $X(\varrho)$, the invariant measure $\mu$ supported by this orbit lies in $\mathcal{M}_{X(\varrho)}$, and clearly $\mu\left(\langle m\rangle_{X(\varrho)}\right)=1 / 2$. But by Remark 3.14, $S_{\varrho}\left(\langle m\rangle_{X(\varrho)}\right)=\{\varrho\}=\pi /(a \pi+1)<1 / 2$. Therefore $S_{\varrho}$ does not dominate $\mu$, and the proof is complete.

\section{Beta-shifts with a largest invariant measure}

Having identified, in Section 4, certain Sturmian-type beta-shifts which do not have a largest invariant measure, we now show that all other Sturmian-type beta-shifts do have a largest invariant measure.

Firstly, the singleton beta-shift $\{\overline{0}\}$ is easily dealt with: it supports a unique (invariant) probability measure $\delta_{\overline{0}}$, which of course is its largest invariant measure. Henceforth, therefore, we shall assume that $X \in \mathfrak{B} \backslash\{\overline{0}\}$.

Notation 5.1. Let $\varrho=A+\pi / q$ with $A, \pi \in \mathbb{N}_{0}, q \in \mathbb{N}, \operatorname{gcd}(\pi, q)=1$. Let $s_{1}<\ldots<s_{q}$ denote the atoms of $S_{X(\varrho)}$, and define

$$
J_{0}:=\left[\overline{0}, s_{1}\right]_{X(\varrho)}, \quad \text { and } \quad J_{j}:=\left(s_{j}, s_{j+1}\right]_{X(\varrho)} \quad \text { for } 1 \leq j \leq q-1 .
$$

The collection $\left\{J_{i}\right\}_{i=0}^{q-1}$ will be called the Sturmian partition of $X(\varrho)$.

With the notation as above, $J_{1} \cup \ldots \cup J_{q-\pi-1} \subset\left\langle m_{X(\varrho)}-1\right\rangle_{X(\varrho)}$, and $J_{q-\pi} \cup \ldots \cup J_{q-1}=$ $\left\langle m_{X(\varrho)}\right\rangle_{X(\varrho)}$.

Notation 5.2. Henceforth it will be notationally convenient to denote $\left[x, x^{\prime}\right]_{X}$ by $\left[x, x^{\prime}\right]$, and $\left(x, x^{\prime}\right]_{X}$ by $\left(x, x^{\prime}\right]$.

The following Lemma 5.3 records the mapping properties of the Sturmian partition of $X(\varrho)$ under the shift map $\sigma: X(\varrho) \rightarrow X(\varrho)$. A preimage always means a preimage under $\left.\sigma\right|_{X(\varrho)}$; so to say that $x$ has $l$ preimages means that $\left(\left.\sigma\right|_{X(\varrho)}\right)^{-1}(x)$ has cardinality $l$. The key feature is that the last $\pi$ intervals $J_{q-\pi}, \ldots, J_{q-1}$ are mapped onto the first $\pi$ intervals $J_{0}, \ldots, J_{\pi-1}$, thus points in these first $\pi$ intervals have one more shift preimage than other points in $X(\varrho)$ :

Lemma 5.3. For $\varrho=A+\pi / q$ with $A, \pi \in \mathbb{N}_{0}, q \in \mathbb{N}, \operatorname{gcd}(\pi, q)=1$, let $J_{0}, \ldots, J_{q-1}$ be the Sturmian partition of $X(\varrho)$.

(a) Each $x \in J_{0} \cup \ldots \cup J_{\pi-1}$ has $m_{X(\varrho)}+1$ preimages, of which $m_{X(\varrho)}$ lie in $J_{0}$, and one lies in $X(\varrho) \backslash J_{0}$. Each $x \in J_{n}, n \in[0, \pi-1]$, has a preimage in $J_{n+q-\pi}$.

(b) Each $x \in J_{\pi}$ has $m_{X(\varrho)}$ preimages, all of which lie in $J_{0}$.

(c) Each $x \in J_{\pi+1} \cup \ldots \cup J_{q-1}$ has $m_{X(\varrho)}$ preimages, of which $m_{X(\varrho)}-1$ lie in $J_{0}$, and one lies in $X(\varrho) \backslash J_{0}$. Each $x \in J_{n}, n \in[\pi+1, q-1]$, has a preimage in $J_{n-\pi}$.

Proof. Let us denote $X(\varrho)$ by $X$, and $m_{X(\varrho)}$ by $m$. Points in $J_{0} \cup \ldots \cup J_{\pi-1}=\left[\overline{0}, \sigma\left(X^{+}\right)\right]$ have precisely $m+1$ preimages, and points in $J_{\pi} \cup \ldots \cup J_{q-1}=\left(\sigma\left(X^{+}\right), X^{+}\right]$have precisely $m$ preimages (cf. Remark 2.17).

(a) Let $n \in[0, \pi-1]$. Now $J_{n}=\left(s_{n}, s_{n+1}\right]$, so

$$
m J_{n}=\left(s_{n+q-\pi}, s_{n+1+q-\pi}\right]=J_{n+q-\pi} \subset J_{1} \cup \ldots \cup J_{q-\pi-1} \subset X \backslash J_{0},
$$


thus each $x \in J_{0} \cup \ldots \cup J_{\pi-1}$ has at least one preimage in $X \backslash J_{0}$. But $x$ has at most one preimage in $X \backslash J_{0}$, since $X \backslash J_{0}$ is a proper sub-interval of $\left[X^{-}, X^{+}\right]$, so each $x \in J_{0} \cup \ldots \cup J_{\pi-1}$ has exactly one preimage in $X \backslash J_{0}$, and hence exactly $m$ preimages in $J_{0}$.

(b) If $\varrho \in \mathbb{N}_{0}$ then $\pi=0$ and $m=\varrho$, so $J_{\pi}=J_{0}=\left[\overline{0}, s_{1}\right]=\left[\overline{0}, X^{+}\right]=X=F_{\varrho}$, so every point in $J_{\pi}$ has precisely $\varrho=m$ preimages in $X=J_{0}$, as required. If $\varrho \notin \mathbb{N}_{0}$ then $\pi \geq 1$ and $J_{\pi}=\left(s_{\pi}, s_{\pi+1}\right]$, so $\left(m_{X}-1\right) J_{\pi}=\left(\left(m_{X}-1\right) s_{\pi}, s_{1}\right] \subset J_{0}$. Moreover, if $x \in J_{\pi}$ and $k \in\left[0, m_{X}\right)$ then $k x<\left(m_{X}-1\right) x$, so $k x \in J_{0}$. So if $x \in J_{\pi}$ then all $m_{X}$ of its preimages lie in $J_{0}$.

(c) Let $n \in[\pi+1, q-1]$. Now $J_{n}=\left(s_{n}, s_{n+1}\right]$, so

$$
(m-1) J_{n}=\left(s_{n-\pi}, s_{n+1-\pi}\right]=J_{n-\pi} \subset J_{1} \cup \ldots \cup J_{q-\pi-1} \subset X \backslash J_{0},
$$

so each $x \in J_{\pi+1} \cup \ldots \cup J_{q-1}$ has at least one preimage in $X \backslash J_{0}$. But $x$ has at most one preimage in $X \backslash J_{0}$, since $X \backslash J_{0}$ is a proper sub-interval of $\left[X^{-}, X^{+}\right]$, so each $x \in$ $J_{\pi+1} \cup \ldots \cup J_{q-1}$ has exactly one preimage in $X \backslash J_{0}$, and hence exactly $m-1$ preimages in $J_{0}$.

THEOREM 5.4. If $\varrho \in U$ then $\left(\mathcal{M}_{X(\varrho)}, \prec\right)$ has a largest element, namely its Sturmian measure $S_{X(\varrho)}=S_{\varrho}$.

Proof. Let $X=X(\varrho)$ where $\varrho \in U$. So $\varrho=A+\frac{\pi}{q}$ where $\pi \in \mathbb{N}_{0}$ and either $A \in \mathbb{N}$, $q=\pi+1$, or $A=0, q=a \pi+1$ for some $a \in \mathbb{N}$. We must show that $\mu \prec S_{X}$ for every $\mu \in \mathcal{M}_{X}$. By Lemma 2.10, this is true if and only if $\mu\left(x, X^{+}\right] \leq S_{X}\left(x, X^{+}\right]$for all $x \in X$. But $S_{X}$ is purely atomic, with atoms $s_{1}<\ldots<s_{q}$, so it is enough to show that

$$
\mu\left(s_{q-i}, X^{+}\right] \leq S_{X}\left(s_{q-i}, X^{+}\right]
$$

for all $1 \leq i \leq q-1$. In other words, we wish to show that $S_{X}$ is a measure of maximum hitting frequency (cf. Remark 2.12 (b)) for each interval $\left(s_{q-i}, X^{+}\right]=J_{q-i} \cup \ldots \cup J_{q-1}$, $1 \leq i \leq q-1$. The main substance of our argument will be to first establish (18) for $i \in[1, \pi]$. Having proved this, we will then explain how the case of general $1 \leq i \leq q$ follows.

Therefore, for each $i \in[1, \pi]$, define

$$
f_{i}:=\chi_{\left(s_{q-i}, s_{q}\right]} .
$$

Define $\varphi_{i}: X \rightarrow \mathbb{R}$ to be constant on each interval $J_{j}$ of the Sturmian partition, with

$$
\left.\varphi_{i}\right|_{J_{0}}:=0 \text {, }
$$

and for $l \in[0, a-1]$,

$$
\left.\varphi_{i}\right|_{J_{k+l \pi}}:= \begin{cases}\frac{i}{q}(a k-l) & \text { for } k \in[1, \pi-(i+1)] \\ \frac{i}{q}(a k-l)+\pi-k-i & \text { for } k \in[\pi-i, \pi] .\end{cases}
$$

Note that in fact, for $l \in[0, a-1]$,

$$
\left.\varphi_{i}\right|_{J_{k+l \pi}}=\frac{i}{q}(a k-l) \text { for all } k \in[1, \pi-i] .
$$

In order to prove (18) we first claim that

$$
\max _{y \in \sigma^{-1}(x)}\left(f_{i}+\varphi_{i}\right)(y)=\varphi_{i}(x)+\frac{i}{q} \quad \text { for all } x \in X .
$$

For each $x \in X$, let $\tau(x)$ denote its unique preimage in the interval $\left(X^{-}, X^{+}\right]$; that is, $\tau(x)=m_{X} x$ for $x \in\left[\overline{0}, \sigma\left(X^{+}\right)\right]$, and $\tau(x)=\left(m_{X}-1\right) x$ for $x \in\left(\sigma\left(X^{+}\right), X^{+}\right]$(cf. Remark 2.17). As a step towards establishing (21) we shall first prove that for all $x \in X$,

$$
\max _{y \in \sigma^{-1} x}\left(f_{i}+\varphi_{i}\right)(y)=\left(f_{i}+\varphi_{i}\right)(\tau(x))
$$

Note that (22) is trivially true if $x \in J_{\pi}$, since all its preimages lie in $J_{0}$ by Lemma 5.3 (b), hence $f_{i}+\varphi_{i}$ vanishes at each such preimage. 
Now suppose $\varrho=A+\pi / q$, where $A, \pi \in \mathbb{N}_{0}$, and $q=a \pi+1$ for $a \in \mathbb{N}$; at this stage we do not require the fact that if $A>0$ then $a=1$. Every $x \in J_{0} \cup \ldots \cup J_{\pi-1}$ has, by Lemma 5.3 (a), at least one preimage in $J_{0}$, and precisely one preimage in $X \backslash J_{0}$, namely $\tau(x)$. Since $\left.\left(f_{i}+\varphi_{i}\right)\right|_{J_{0}}=0$, to establish $(22)$ it suffices to prove that $\left(f_{i}+\varphi_{i}\right)(\tau(x))>0$. For this, let $n \in[0, \pi-1]$ be such that $x \in J_{n}$, so that $\tau(x) \in J_{n+q-\pi}$ by Lemma 5.3 (a). We wish to show that

$$
\left.\left(f_{i}+\varphi_{i}\right)\right|_{J_{n+q-\pi}}>0 .
$$

Firstly, if $n \in[0, \pi-(i+1)]$ then $n+q-\pi \in[q-\pi, q-(i+1)] \subset[0, q-(i+1)]$, so $\left.f_{i}\right|_{J_{n+q-\pi}}=0$, and therefore (19), (20) give

$$
\left.\left(f_{i}+\varphi_{i}\right)\right|_{J_{n+q-\pi}}=\left.\varphi_{i}\right|_{J_{n+q-\pi}}=\left.\varphi_{i}\right|_{J_{(n+1)+(a-1) \pi}}=\frac{i}{q}(\text { an }+1)>0 .
$$

Secondly, if $n \in[\pi-i, \pi-1]$ then $n+q-\pi \in[q-i, q-1]$, so $\left.f_{i}\right|_{J_{n+q-\pi}}=1$, and therefore

$$
\begin{aligned}
\left.\left(f_{i}+\varphi_{i}\right)\right|_{J_{n+q-\pi}} & =1+\left.\varphi_{i}\right|_{J_{n+q-\pi}} \\
& =1+\left.\varphi_{i}\right|_{J_{(n+1)+(a-1) \pi}} \\
& =\frac{i}{q}(\text { an }+1)+\pi-n-i \\
& =(a i / q-1) n+\pi-i+i / q .
\end{aligned}
$$

The expression (25) is decreasing in $n$, because $a i / q \leq a \pi / q<1$, so for $n \in[\pi-i, \pi-1]$ it attains its minimum when $n=\pi-1$. That is,

$$
\left.\left(f_{i}+\varphi_{i}\right)\right|_{J_{n+q-\pi}} \geq\left(\frac{a i}{q}-1\right)(\pi-1)+\pi-i+\frac{i}{q}=1-\frac{a i}{q} \geq 1-\frac{a \pi}{q}>0 .
$$

Combining (24) and (26) yields (23), and hence (22) holds for all $x \in J_{0} \cup \ldots \cup J_{\pi-1}$.

If $\varrho \in[1, \infty) \cap U$ then $q=\pi+1$, so the preceding argument establishes (22) for all $x \in J_{0} \cup \ldots \cup J_{q-1}=X$, as required. It therefore remains to check that (22) holds when $\varrho \in[0,1) \cap U$ and $x \in J_{\pi+1} \cup \ldots \cup J_{q-1}$. But in this case $m_{X}=1$, so Lemma 5.3 (c) implies that $x$ has a single preimage, namely $\tau(x)$, and thus (22) is trivially true. Therefore, for all $\varrho \in U$, we have established (22) for all $x \in X$.

We shall now derive (21) from (22) by checking that, for all $x \in X$,

$$
\left(f_{i}+\varphi_{i}\right)(\tau(x))=\varphi_{i}(x)+\frac{i}{q}
$$

If $x \in J_{\pi}$ then $\left(f_{i}+\varphi_{i}\right)(\tau(x))=0$ since $\tau(x) \in J_{0}$, and $\varphi_{i}(x)=-i / q(k=\pi, l=0$ in (19)), so (27) holds. If $x \in J_{n}$ for $n \in[0, \pi-(i+1)]$ then again by (19), $\varphi_{i}(x)=i a n / q$, so $\varphi_{i}(x)+i / q=i(a n+1) / q=\left(f_{i}+\varphi_{i}\right)(\tau(x))$ by $(24)$, so (27) holds. If $x \in J_{n}$ for $n \in[\pi-i, \pi-1]$ then by (19), $\varphi_{i}(x)=i a n / q+\pi-n-i$, so $\varphi_{i}(x)+i / q=i(a n+1) / q+\pi-n-i=\left(f_{i}+\varphi_{i}\right)(\tau(x))$ by (25), so (27) holds. As before, if $\varrho \in[1, \infty) \cap U$ then $q=\pi+1$ so (27) holds for all $x \in X$. Finally, if $\varrho \in[0,1) \cap U$ (i.e. $m_{X}=1$ ) and $x \in J_{\pi+1} \cup \ldots \cup J_{q-1}$ then $x \in J_{k+l \pi}$ for some $k \in[1, \pi], l \in[1, a-1]$. Now by Lemma $5.3(\mathrm{c}), \tau(x) \in J_{k+(l-1) \pi}$, so $f_{i}(\tau(x))=0$, therefore $\left(f_{i}+\varphi_{i}\right)(\tau(x))=\varphi_{i}(\tau(x))=\left.\varphi_{i}\right|_{J_{k+(l-1) \pi}}=\varphi_{i}(x)+i / q$, so $(27)$ holds. We have therefore proved that (27) holds for all $x \in X$, and combined with (22) this yields (21).

We can now derive (18) from (21) as follows. Replacing $x$ by $\sigma(x)$ in (21) yields

$$
\left(f_{i}+\varphi_{i}\right)(x) \leq \max _{y \in \sigma^{-1} \sigma(x)}\left(f_{i}+\varphi_{i}\right)(y)=\varphi_{i}(\sigma(x))+\frac{i}{q} \quad \text { for all } x \in X,
$$

so the function $f_{i}+\varphi_{i}-\varphi_{i} \circ \sigma$ is bounded above by $i / q$. Therefore for all $\mu \in \mathcal{M}_{X}$,

$$
\mu\left(s_{q-i}, X^{+}\right]=\mu\left(f_{i}\right)=\mu\left(f_{i}+\varphi_{i}-\varphi_{i} \circ \sigma\right) \leq \frac{i}{q} .
$$

But $S_{X}\left(s_{q-i}, X^{+}\right]=i / q$, so (18) follows, in the case $i \in[1, \pi]$. 
We now show how to deduce (18) for general $i \in[1, q-1]$. Writing $i=b \pi+j$ for $b \in[0, a-1], j \in[1, \pi]$, we first claim that

$$
\mu\left(s_{q-(b \pi+j)}, s_{q-b \pi}\right] \leq S_{X}\left(s_{q-(b \pi+j)}, s_{q-b \pi}\right] \quad \text { for all } \mu \in \mathcal{M}_{X} .
$$

The proof of (28) will be via induction on $b$; note that the base case $b=0, j \in[1, \pi]$, has already been established (it is precisely (18) for $i \in[1, \pi]$ ). To prove (28) for $b \in[1, a-1]$, $j \in[1, \pi]$, note that $\sigma\left(\left(s_{q-(b \pi+j)}, s_{q-b \pi}\right]\right)=\left(s_{q-((b-1) \pi+j)}, s_{q-(b-1) \pi}\right]$, so

$$
\left(s_{q-(b \pi+j)}, s_{q-b \pi}\right] \subset \sigma^{-1}\left(s_{q-((b-1) \pi+j)}, s_{q-(b-1) \pi}\right] .
$$

Therefore, if $\mu \in \mathcal{M}_{X}$ then

$$
\mu\left(s_{q-(b \pi+j)}, s_{q-b \pi}\right] \leq \mu\left(\sigma^{-1}\left(s_{q-((b-1) \pi+j)}, s_{q-(b-1) \pi}\right]\right)=\mu\left(s_{q-((b-1) \pi+j)}, s_{q-(b-1) \pi}\right] .
$$

But

$$
\sigma^{-1}\left(s_{q-((b-1) \pi+j)}, s_{q-(b-1) \pi}\right]=\left(s_{q-(b \pi+j)}, s_{q-b \pi}\right] \cup Y
$$

where $Y \subset J_{0} \subset\left[\overline{0}, X^{-}\right]$, and the support of $S_{X}$ is contained in $\left(X^{-}, X^{+}\right]$(cf. Remark 3.8), so

$$
S_{X}\left(s_{q-(b \pi+j)}, s_{q-b \pi}\right]=S_{X}\left(\sigma^{-1}\left(s_{q-((b-1) \pi+j)}, s_{q-(b-1) \pi}\right]\right)=S_{X}\left(s_{q-((b-1) \pi+j)}, s_{q-(b-1) \pi}\right] .
$$

Combining the inductive hypothesis

$$
\mu\left(s_{q-((b-1) \pi+j)}, s_{q-(b-1) \pi}\right] \leq S_{X}\left(s_{q-((b-1) \pi+j)}, s_{q-(b-1) \pi}\right]
$$

with (29) and (30) yields (28), as claimed.

We now use (28) to deduce (18), i.e. that $S_{X}$ is a measure of maximum hitting frequency for the interval $\left(s_{q-i}, s_{q}\right]$, in the remaining cases $i \in[\pi+1, q-1]$. Writing $i=c \pi+\kappa$, where $\kappa \in[1, \pi], c \in[1, a-1]$, we can write $\left(s_{q-i}, s_{q}\right]$ as the disjoint union

$$
\left(s_{q-i}, s_{q}\right]=\left(s_{q-(c \pi+\kappa)}, s_{q-c \pi}\right] \cup \bigcup_{\lambda=1}^{c}\left(s_{q-\lambda \pi}, s_{q-(\lambda-1) \pi}\right] .
$$

Now $S_{X}$ is a measure of maximum hitting frequency for each of the $c+1$ intervals in the disjoint union (32), using (28) in the cases $(b, j)=(\lambda-1, \pi)$ for $\lambda \in[1, c]$, and in the case $(b, j)=(c, \kappa)$. Consequently, $S_{X}$ is also a measure of maximum hitting frequency for the interval $\left(s_{q-i}, s_{q}\right]$, as required.

REMARK 5.5. The proof of Theorem 5.4 via the introduction of the function $\varphi_{i}$ is inspired by ideas of Bousch [7] in a related setting, in particular his notion of the Sturmian condition (cf. also $[\mathbf{3}, \mathbf{1 6}]$ ). The usefulness in ergodic optimization of solutions $\varphi_{i}$ to equations of the form (21) had been observed by Conze \& Guivarc'h [11].

It is now a simple matter to complete the theorems announced in Section 1:

Proof of Theorems 1.1-1.3. In view of Theorems 4.5 and 5.4, to conclude the proof of Theorems 1.1-1.3 it only remains to check the equivalence of statements (ii) and (iii) in Theorems 1.2 and 1.3. Let $X$ denote the beta-shift whose largest element $X^{+}$is the periodic sequence with periodic word $\left(10^{a-1}\right)^{\pi} 0$ for $a, \pi \geq 1$ (respectively $B^{\pi}(B-1)$ for some $\pi \geq 1$, $B \geq 2$ ). By [6, Prop. $2.3(2)], X=X_{\beta}$ if and only if $1=\sum_{i=1}^{\infty}\left(X^{+}\right)_{i} \beta^{-i}$, and this equation is easily seen to be equivalent to $\zeta^{a \pi+1}-\sum_{i=0}^{\pi} \zeta^{i a}=0$ (respectively $\zeta^{\pi+1}-B \sum_{i=0}^{\pi} \zeta^{i}=0$ ).

\section{Acknowledgments}

The first author was partially supported by an EPSRC PhD studentship and an Erwin Schrödinger Institute Junior Research Fellowship. The second author was partially supported by an EPSRC Advanced Research Fellowship. 


\section{References}

[1] C. D. Aliprantis \& K. C. Border, Infinite dimensional analysis: a hitchhiker's guide, 2nd edition, SpringerVerlag, Berlin, 1999.

[2] J.-P. Allouche \& J. Shallit, Automatic sequences. Theory, applications, generalizations, Cambridge University Press, Cambridge, 2003.

[3] V. Anagnostopoulou, K. Díaz-Ordaz, O. Jenkinson \& C. Richard, Escape from a semi-circle and Sturmian maximizing measures, preprint, 2007.

[4] J. Berstel \& P. Séébold, Sturmian words, in: M. Lothaire, Algebraic combinatorics on words, Encyclopaedia of mathematics and its applications, vol. 90, Cambridge University Press, 2002, pp. 45-110.

[5] A. Bertrand-Mathis, Développements en base $\theta$ et répartition modulo 1 de la suite $\left(x \theta^{n}\right)$, Bull. Soc. Math. Fr., 114 (1986), 271-324.

[6] F. Blanchard, $\beta$-expansions and symbolic dynamics, Theoret. Comput. Sci., 65 (1989), 131-141.

[7] T. Bousch, Le poisson n'a pas d'arêtes, Ann. Inst. Henri Poincaré (Proba. et Stat.), 36 (2000), 489-508.

[8] T. Bousch \& J. Mairesse, Asymptotic height optimization for topical IFS, tetris heaps, and the finiteness conjecture, Jour. Amer. Math. Soc., 15 (2002), 77-111.

[9] S. Bullett \& P. Sentenac, Ordered orbits of the shift, square roots, and the devil's staircase, Math. Proc. Camb. Phil. Soc., 115 (1994), 451-481.

[10] D. P. Chi \& D. Y. Kwon, Sturmian words, $\beta$-shifts, and transcendence, Theoret. Comput. Sci., 321 (2004), 395-404.

[11] J.-P. Conze \& Y. Guivarc'h, Croissance des sommes ergodiques et principe variationnel, manuscript, 1993.

[12] K. Dajani \& C. Kraaikamp, Ergodic theory of numbers, Carus Mathematical Monographs 29, Mathematical Association of America, Washington, DC, 2002.

[13] J. Dufresnoy \& Ch. Pisot, Sur un ensemble fermé d'entiers algébriques, Ann. Sci. ENS, 70 (1953), 105133.

[14] Ch. Frougny \& B. Solomyak, Finite beta-expansions, Ergod. Th. E Dyn. Sys., 12 (1992), 713-723.

[15] G. H. Hardy \& E. M. Wright, An introduction to the theory of numbers, 5th edition, Oxford University Press, 1979.

[16] E. Harriss \& O. Jenkinson, Flattening functions on flowers, Ergod. Th. \& Dyn. Sys., 27 (2007), $1865-1886$.

[17] F. Hofbauer, $\beta$-shifts have unique maximal measure, Monatsh. Math., 85 (1978), 283-300.

[18] O. Jenkinson, Conjugacy rigidity, cohomological triviality, and barycentres of invariant measures, Ph.D. thesis, University of Warwick, 1996.

[19] O. Jenkinson, Frequency locking on the boundary of the barycentre set, Experimental Mathematics, 9 (2000), 309-317.

[20] O. Jenkinson, Maximum hitting frequency and fastest mean return time, Nonlinearity, 18 (2005), 23052321.

[21] O. Jenkinson, Ergodic optimization, Discrete 86 Cont. Dyn. Sys., 15 (2006), 197-224.

[22] O. Jenkinson, Optimization and majorization of invariant measures, Electron. Res. Announc. Amer. Math. Soc., 13 (2007), 1-12.

[23] O. Jenkinson, A partial order on $\times 2$-invariant measures, Math. Res. Lett., to appear.

[24] K. Johnson, Beta-shift dynamical systems and their associated languages, Ph.D. thesis, University of North Carolina, 1999.

[25] T. Kamae, U. Krengel \& G. L. O'Brien, Stochastic inequalities on partially ordered spaces, Ann. Prob., 5 (1977), 899-912.

[26] D. Y. Kwon, A devil's staircase from rotations and irrationality measures for Liouville numbers, Math. Proc. Camb. Phil. Soc., to appear.

[27] A. W. Marshall \& I. Olkin, Inequalities: theory of majorization and its applications, Mathematics in science and engineering vol. 143, Academic Press, 1979.

[28] M. Morse \& G. A. Hedlund, Symbolic Dynamics II. Sturmian Trajectories, Amer. J. Math., 62 (1940), $1-42$.

[29] E. Olivier, N. Sidorov \& A. Thomas, On the Gibbs properties of Bernoulli convolutions related to $\beta$ numeration in multinacci bases, Monatsh. Math., 145 (2005), 145-174.

[30] W. Parry, On the $\beta$-expansions of real numbers, Acta Math. Acad. Sci. Hungar., 11 (1960), 401-416.

[31] K. Petersen, Some Sturmian symbolic dynamics, http://www.math.unc.edu/Faculty/petersen

[32] N. Pytheas Fogg, Substitutions in dynamics, arithmetics and combinatorics, Springer Lecture Notes in Mathematics vol. 1794, 2002.

[33] A. Rényi, Representations of real numbers and their ergodic properties, Acta. Math. Acad. Sci. Hungar., 8 (1957), 477-493.

[34] K. Schmidt, On periodic expansions of Pisot numbers and Salem numbers, Bull. London Math. Soc., 12 (1980), 269-278. 
[35] N. Sidorov, Arithmetic dynamics, in 'Topics in Dynamics and Ergodic Theory', LMS Lecture Notes Ser. 310, pp. 145-189, 2003.

[36] C. Siegel, Algebraic integers whose conjugates lie in the unit circle, Duke Math. J., 11 (1944), 597-602.

[37] K. Sigmund, On the distribution of periodic points for $\beta$-shifts, Monatsh. Math., 82 (1976), $247-252$.

[38] M. Smorodinsky, $\beta$-automorphisms are Bernoulli shifts, Acta Math. Acad. Sci. Hung., 24 (1973), $273-278$.

[39] P. Walters, An introduction to ergodic theory, Springer-Verlag, New York, 1982.

Vasso Anagnostopoulou; School of Mathematical Sciences, Queen Mary, University of LonDon, Mile End RoAd, London, E1 4NS, UK.

vaa@maths.qmul.ac.uk

Oliver Jenkinson; School of Mathematical Sciences, Queen Mary, University of London, Mile End RoAd, London, E1 4NS, UK.

omj@maths . qmul. ac.uk

ww. maths.qmul.ac.uk/ omj 